\title{
Phenylethylamine, a possible link to the antidepressant effects of exercise?
}

\author{
A Szabo, E Billett, J Turner
}

\begin{abstract}
Objectives-To determine in this pilot study whether aerobic exercise affects phenylacetic acid concentration in the urine.

Methods-Twenty healthy men provided 24 hour urine samples on two consecutive days for the determination of phenylacetic acid levels. Before and during day 1, subjects refrained from physical activity; on day 2 subjects ran on a treadmill at $70 \%$ of their maximal heart rate reserve (MHRR) for 30 minutes.

Results-The 24 hour mean urinary concentration of phenylacetic acid was increased by $77 \%$ after exercise.

Conclusion-As phenylacetic acid concentration in urine reflects phenylethylamine level, which is known to have antidepressant effects, phenylethylamine may be linked to the therapeutic effects of physical exercise on depression. (Br F Sports Med 2001;35:342-343)
\end{abstract}

Keywords: depression; exercise; phenylacetic acid; phenylethylamine

The current consensus is that physical activity has antidepressant effects. ${ }^{1}$ Indeed, doctors widely recommend exercise either as treatment for mild depression or as complementary treatment to drug and/or psychotherapy in cases of more severe depression. ${ }^{1}$ The mode of action of exercise, however, remains unclear.

Phenylethylamine is an endogenous neuroamine that has been linked to the regulation of physical energy, mood, and attention. ${ }^{2}$ Monoamine oxidase B selectively metabolises phenylethylamine to phenylacetic acid. There is evidence to indicate that levels of phenylethylamine and phenylacetic acid are very low in the biological fluids of depressed patients. ${ }^{3}$ As phenylethylamine turnover is very fast and phenylacetic acid levels in the biological fluids are far higher than phenylethylamine levels, it has been suggested that phenylacetic acid excretion is a better measure than phenylethylamine for examining the modulatory role of phenylethylamine. Studies on urinary excretion of phenylacetic acid have shown that about $60 \%$ of unipolar and bipolar patients have lower than normal levels. ${ }^{2}$ Administration of phenylethylamine or its precursor L-phenylalanine, in conjunction with selegiline, a selective monoamine oxidase $\mathrm{B}$ inhibitor, has been reported to alleviate depression and to produce improvements in mood. The effects are sustained and also apparent in some patients who are insensitive to conventional treatment. $^{23}$

In view of the links between exercise and depression, and phenylethylamine and depression, the relation between exercise and phenylethylamine also deserves attention. Furthermore, phenylethylamine is involved in the modulation of noradrenergic and dopaminergic synapses. ${ }^{2}$ In its role as an inhibitor of noradrenergic reuptake, phenylethylamine may be implicated in physical exercise. Considering that there is a dynamic equilibrium between central and peripheral phenylethylamine, because of its high lipid solubility and easy passage through the blood/brain barrier, examination of the relation between phenylethylamine (as measured by urinary phenylacetic acid levels) and exercise is further warranted. To the best of our knowledge, this study is the first attempt to test the effects of exercise on phenylacetic acid levels.

\section{Methods}

Twenty healthy male volunteers (mean (SD) age 22.1 (4.1) years) agreed to be tested by signing an informed consent form. Their mean (SD) body mass index was 23.5 (1.6), their mean (SD) resting heart rate was $64(7.8)$ beats/min, and every week they exercised for 2.6 (1.4) hours aerobically and 1.4 (1.3) hours anaerobically. The subjects refrained from exercise for 24 hours (day 0) before the experiment. Urine was then collected by the volunteers for a 24 hour control non-exercising period (day 1). On day 2, the participants ran on a treadmill (Powerjog JX100) at $70 \%$ of their maximal hear rate reserve (MHRR) for 30 minutes in the laboratory. This exercise intensity was selected because a recent literature review $^{4}$ shows that changes in mood are commonly reported at $60-80 \%$ MHRR. On completion of the exercise and before recovery, subjects were asked to indicate the perceived intensity of their workout on a three point (light, moderate, hard) rating scale. After the laboratory session, the participants collected their urine for a further 24 hours (day 2). Once collected, the urine was kept at $4^{\circ} \mathrm{C}$ until transported to the laboratory where it was frozen. Phenylacetic acid levels in the samples were stable using this protocol. Urinary volumes were all in excess of 0.8 litres. $^{5}$ As the total weight of phenylacetic acid in the 24 hour urine was measured ( $\mathrm{mg} / 24$ hours), the subjects were free to consume water or other liquids ad libitum.

The concentrations of urinary phenylacetic acid were determined by the gas liquid chromatography method of Gusovsky et al. ${ }^{5}$. 


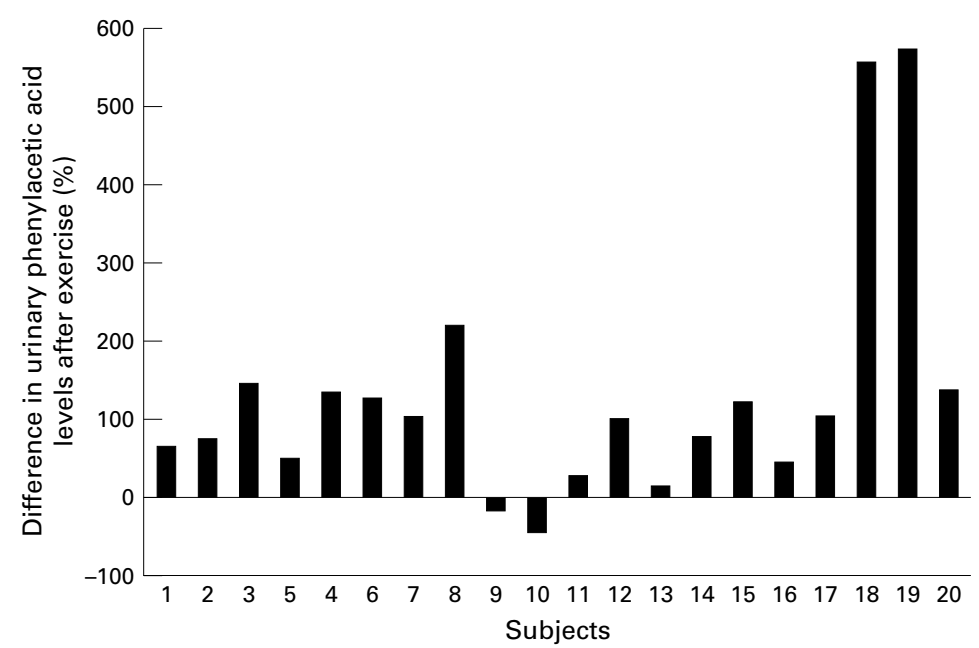

Figure 1 Percentage difference in urinary phenylacetic acid after exercise. Phenylacetic acid concentrations were measured as $m g / 24$ hours and percentages are compared with values obtained before exercise.
Each sample was run in the presence of a standard concentration of an internal control, phenylpropionic acid. Standards of phenylacetic acid in the range $10-40 \mu \mathrm{g} / \mathrm{ml}$ were used to calibrate the column. Peak areas of phenylacetic acid/ phenylpropionic acid were calculated and used in the analyses.

\section{Results}

In 18 of the 20 subjects, the level of phenylacetic acid in the urine was higher after exercise, increasing by $14-572 \%$ compared with the values before exercising (fig 1). The mean (SD) value of phenylacetic acid before exercise was 99.4 (54.4) $\mathrm{mg} / 24$ hours and 176.0 (47.7) $\mathrm{mg} / 24$ hours after exercise. The difference between the two measurements was significant $\left(F_{1,19}=26.6, \mathrm{p}<0.0001\right.$; effect size $(E S)=1.2)$. The correlation between the two sets of scores, however, was not significant $(r=0.33, \mathrm{p}=0.09)$.

\section{Discussion}

These results show substantial increases in urinary phenylacetic acid levels 24 hours after moderate to high intensity aerobic exercise. As phenylacetic acid reflects phenylethylamine levels ${ }^{3}$, and the latter has antidepressant effects, the antidepressant effects of exercise appear to be linked to increased phenylethylamine concentrations. Furthermore, considering the structural and pharmacological analogy between amphetamines and phenylethylamine, it is conceivable that phenylethylamine plays a role in the commonly reported "runners high" thought to be linked to cerebral $\beta$-endorphin activity. The substantial increase in phenylacetic acid excretion in this study implies that phenylethylamine levels are affected by exercise.

Although about $75 \%$ of subjects responded relatively homogeneously, there was considerable interindividual variability in the phenylacetic acid responses to exercise (fig 1). Interestingly, 17 of the subjects rated the exercise level as moderate, whereas three (11, 18 , and 19 in fig 1) rated it as hard. Two of the latter (subjects 18 and 19) also showed the most noticeable increase in phenylacetic acid in the following 24 hours. (It should be noted that our statistical conclusions would not change if the outlier cases, 18 and 19, were disregarded.) The lack of significant correlation between phenylacetic acid levels before and after exercise indicates that the former only accounted for about $11 \%\left(r=0.33 ; r^{2}=0.11\right)$ of the changes in the latter. Consequently, many factors may mediate phenylacetic acid responses to exercise, possibly including perceived and/or actual exercise intensity. Determination of these factors remains the object of future inquiries.

The present findings should serve as an incentive for further research into the mechanism(s) linking phenylethylamine to exercise. Such research should consider some important factors that were not addressed in this pilot study. Firstly, the inclusion of a passive activity control group is advised. Secondly, instead of relying on MHRR as here, future studies need to assess the actual $\mathrm{VO}_{2} \mathrm{MAX}$ of the participants. Thirdly, the changes in phenylacetic acid may be different in a sedentary sample in contrast with the relatively fit and physically active sample tested here. Therefore the influence of fitness on phenylacetic acid levels also needs to be examined. Finally, the effects reported here should also be examined in a clinically depressed population.

1 Mutrie N. The relationship between physical activity and clinically defined depression. In: Biddle S, Fox K, Boutcher S, eds. Physical activity and psychological well-being. London: Routledge, 2000:46-62.

2 Sabelli H, Fink P, Fawcett J, et al. Sustained antidepressant effects of PEA replacement. 7 Neuropsychiatry 1996;8:168 71.

3 Sabelli H, Javaid J. Phenylethlyamine modulation of affect: therapeutic and diagnostic implications. F Neuropsychiatry Clin Neurosci 1995;7:6-14.

4 Ekkekakis P, Petruzzello S. Acute aerobic exercise and Ekfeckakis P, Petruzzello S. Acute aerobic exercise and
affect: current status, problems and prospects regarding affect: current status, problems and prospects regarding
dose-response. Sports Med 1999;28:337-74.

5 Gusovsky F, Sabelli H, Fawcett J, et al. Gas-liquid chromatographic determination of phenylacetic acid in urine. Anal Biochem 1984;136:202-7.

\section{Take home message}

A 30 minute bout of moderate to high intensity aerobic exercise increases phenylacetic acid levels in healthy regularly exercising men. The findings may be linked to the antidepressant effects of exercise. 